\title{
Negative Purifying Selection Drives Prion and Doppel Protein Evolution
}

\author{
Kyriakos Tsangaras $\cdot$ Sergios-Orestis Kolokotronis • \\ Rainer G. Ulrich $\cdot$ Serge Morand · Johan Michaux • \\ Alex D. Greenwood
}

Received: 6 September 2013/Accepted: 3 July 2014/Published online: 20 July 2014

(C) Springer Science+Business Media New York 2014

\begin{abstract}
The prion protein $(\mathrm{PrP})$ when misfolded into the pathogenic conformer $\mathrm{PrP}^{\mathrm{Sc}}$ is the major causative agent of several lethal transmissible spongiform encephalopathies in mammals. Studies of evolutionary pressure on the corresponding gene using different datasets have yielded conflicting results. In addition, putative PrP or PrP interacting partners with strong similarity to PrP such as the doppel protein have not been examined to determine if the same evolutionary mechanisms apply to prion paralogs or if there are coselected sites that might indicate how and where the proteins interact. We examined several taxonomic groups that contain model organisms of prion diseases focusing on
\end{abstract}

Kyriakos Tsangaras, Sergios-Orestis Kolokotronis are considered joint first authors.

Electronic supplementary material The online version of this article (doi:10.1007/s00239-014-9632-1) contains supplementary material, which is available to authorized users.

K. Tsangaras · A. D. Greenwood ( $₫)$

Department of Wildlife Diseases, Leibniz Institute for Zoo and Wildlife Research, Alfred-Kowalke-Str. 17, 10315 Berlin, Germany

e-mail: greenwood@izw-berlin.de

S.-O. Kolokotronis

Department of Biological Sciences, Fordham University, 441

East Fordham Road, Bronx, NY 10458, USA

R. G. Ulrich

Friedrich-Loeffler-Institut, Institute for Novel and Emerging

Infectious Diseases, Südufer 10,

17493 Greifswald - Insel Riems, Germany

S. Morand

Institut Des Sciences de l'Evolution, CNRS UMR 5554,

Université de Montpellier II, 34095 Montpellier Cedex 05,

France primates, bovids, and an expanded dataset of rodents for selection pressure on the prion gene (PRNP) and doppel gene $(P R N D)$ individually and for coevolving sites within. Overall, the results clearly indicate that both proteins are under strong selective constraints with relaxed selection on amino acid residues connecting $\alpha$-helices 1 and 2 .

Keywords Prion - Doppel $\cdot P R N P \cdot P R N D \cdot$ Purifying selection $\cdot$ Interacting sites

\section{Introduction}

The prion protein $(\operatorname{PrP})$ is the causal factor in a range of transmissible spongiform encephalopathies (TSEs) including Creutzfeld-Jakob Disease (CJD) in humans, bovine spongiform encephalopathy (BSE) in cattle, scrapie in sheep, and chronic wasting disease (CWD) in wild cervids. Common to all prion diseases regardless of affected species

S. Morand

Department of Parasitology, Faculty of Veterinary Sciences,

Kasetsart University, Bangkok, Thailand

J. Michaux

Centre de Biologie et de Gestion des Populations (CBGP),

Campus International de Baillarguet, 34988 Montferrier-le-Lez,

France

J. Michaux

Conservation Genetics Unit, Institute of Botany, University of

Liège, Liege, Belgium 
is the misfolding of the PrP into a proteinase and heat resistant conformer $\mathrm{PrP}^{\mathrm{Sc}}$. Accumulation of this misfolded conformer leads to severe neurodegeneration by an unknown mechanism (Kraus et al. 2013).

The prion gene (PRNP) belongs to a gene family with several known members widespread in vertebrates including the prion gene itself and two conserved genes derived from duplications of the prion gene, doppel (PRND) and shadoo (SPRN) (Premzl and Gamulin 2007). Although exhibiting different but partially overlapping expression profiles, only $P R N P$ and $S P R N$ are expressed in the central nervous system (CNS) in healthy animals. Mis-expression of $P R N D$ is neurotoxic in mouse neuroblastoma cell lines (Silverman et al. 2000). Shadoo appears to be down-regulated during prion diseases suggesting potential interaction with PrP (Westaway et al. 2011). Similarly, it has been suggested that PrP and Dpl interact as agonists with PrP preventing Dpl neurotoxicity (Sakaguchi 2008). Specific deletions of PRNP result in its inability to prevent Dpl neurotoxicity (Sakaguchi 2008). Further evidence for an interaction is that PrP and Dpl coprecipitate from detergent resistant membrane domains of some cell types from rats (Caputo et al. 2010).

Evolutionary analyses of $P R N P$ have demonstrated conflicting results, with the prion gene being under balancing (Mead et al. 2003), purifying (Seabury et al. 2004), and positive selection (Premzl and Gamulin 2009) depending on the dataset. A recent study including representatives of multiple taxa suggested that positive selection was acting on $\mathrm{PrP}$ in various domains and intradomains (Premzl and Gamulin 2009). However, the selection analysis to date has been done on either, relatively small datasets, species specific or order specific data sets and only on the PRNP gene itself and not its paralogs.

To investigate the interspecific evolution of $P R N P$ and $P R N D$, we examined lagomorph, eulipotyph, primate, and bovid sequences from GenBank along with $19 P R N P$ and 21 $P R N D$ sequences from 10 rodent genera and 21 rodent species produced in this study. The bovids, primates, and rodents in particular include animals susceptible to prion diseases, model organisms in prion research or both. Different models were applied to investigate selective pressure on $P R N P$ and $P R N D$ individually and to identify coevolving sites that might indicate interaction sites in the proteins relative to their structure.

\section{Materials and Methods}

\section{Laboratory Procedures}

Twenty-one rodent samples from Europe and Southeast Asia were used for this study. The Southeast Asian samples were collected as part of a larger project involving authors $\mathrm{S}$. Morand and J. Michaux (CERoPath project, "Community
Ecology of Rodents and their Pathogens in a changing environment," http://www.ceropath.org). Apodemus samples were collected by the Conservation Genetics Unit of the University of Liège. The Myodes glareolus sample KS 10/1240 was trapped in October 2009 in Lower Saxony/ Germany. DNA extractions for all the rodent tissue or blood samples were performed using the DNeasy Blood \& Tissue DNA extraction kit (QIAGEN) following the manufacturer's protocol. Polymerase chain reaction for the PRNP and PRND genes was performed in $25 \mu \mathrm{l}$ reactions containing $0.5 \mathrm{U}$ of MyTaq HS polymerase mix (Bioline), $200 \mathrm{nM}$ primers, and $110 \mathrm{ng}$ of DNA template using the following forward $(\mathrm{F})$ and reverse (R) primer sets PrP_F1 (5'-GTTC(C/T)TCATT TTGCAGATCA-3'), Dpl_F1 (5'-CTTTCCCTTGCAGATT CACC-3'), Dpl_R1 (5'-TCACCTCTGTGGCTGCCAGC$\left.3^{\prime}\right)$, Dpl_F2 (5'-CGCCCAGGAGCCTT(C/T)ATCAARC$\left.3^{\prime}\right)$, Dpl_R2 (5'-CACAAT(G/A)AACCAAA(C/T)GAAAC C(C/T)AGCAG- $\left.3^{\prime}\right), \quad$ Dpl_F3 (5'-CCATGAAGAACCG (G/T)(C/G)TGG-3'), and Dpl_R3 (5'-CTC(C/T)GANGCC $\left.\mathrm{AA}(\mathrm{C} / \mathrm{T}) \mathrm{GTGAC}-3^{\prime}\right)$. Thermocycling conditions were $95{ }^{\circ} \mathrm{C}$ denaturation for $3 \mathrm{~min}$ followed by 35 cycles of $95{ }^{\circ} \mathrm{C}$ for $20 \mathrm{~s}, 55^{\circ} \mathrm{C}$ for $20 \mathrm{~s}, 72{ }^{\circ} \mathrm{C}$ for $25 \mathrm{~s}$, with a final extension of $72{ }^{\circ} \mathrm{C}$ for $2 \mathrm{~min}$. Positive PCR amplification products were purified using the QIAquick PCR purification kit (QIAGEN), and sequenced using BigDye chemistry on a 3730 DNA Analyzer (Applied Biosystems). The existence of heterozygotes was examined by inspecting the traces for multiple peaks at a single position. For the species Bandicota savilei, and Rattus argentiventer several (up to three) individual rodents from different populations were available to be sequenced and identical sequences obtained and, though not conclusive for all species, suggested that intraspecific polymorphism did not affect the results.

Sequence Alignment, Phylogenetic, and Selection Analyses

$P R N P$ and PRND GenBank accession codes were as follows: Mus cervicolor (KF466919, KF466939), Mus cookii (KF466920, KF466940), Mus caroli (KF466921, KF46 6941), Mus fragilicauda (KF466956, KF466957), Rattus losea (KF466924, KF466954) Rattus argentiventer (KF466 955, KF466925), Rattus nitidus (KF466938, KF466952), Apodemus sylvaticus (KF466922), Apodemus fulvipectus (KF466923, KF466953), Apodemus mystacinus (KF466 942, KF466935), Leopoldamys sabanus (KF466928, KF4 66943), Leopoldamys edwardsi (KF466944, KF466929), Bandicota indica (KF466930, KF466950), Bandicota savilei (KF466951, KF466931), Saxatilomys paulinae (KF466937, KF466958), Chiropodomys gliroides (KF466 945, KF466932), Berylmys berdmorei (KF466936, KF46 6946), Maxomys surifer (KF466947, KF466933), Myodes glareolus (KF466934). 
Nucleotide sequences were aligned in TranslatorX (Abascal et al. 2010) using MAFFT 7 (Katoh and Standley 2013) and inspected for coding frame-disrupting substitutions. Alignments can be found in the supplemental materials. The phylogenetic relationships among orthologs across species were estimated in a maximum likelihood (ML) framework in the POSIX-Threads built of RAxML 8.0.2 (Stamatakis 2006). Nucleotide sequences were examined with the GTR $+\Gamma_{4}$ (Lanave et al. 1984; Yang 1993) substitution model. Ten maximum likelihood (ML) searches were run starting from a maximum parsimony stepwise-addition tree. Internode branch robustness was evaluated through 500 parametric bootstrap pseudoreplicates (Felsenstein 1981).

The signature of natural selection on both gene sequences was examined using three probabilistic methods implemented in HyPhy 2.1.2 (Pond et al. 2005) and the Datamonkey webserver (http://www.datamonkey.org; (Delport et al. 2010): mixed effects model evolution (MEME) —an extension of the fixed effects likelihood method (Kosakovsky Pond and Frost 2005) allowing $\omega(=\mathrm{d} N / \mathrm{d} S)$ to vary along the phylogeny branches (Murrell et al. 2012), Fast Unconstrained Bayesian AppRoximation (FUBAR)—a new empirical Bayes method for estimating codon-wise trends of negative or positive selection (Murrell et al. 2013), and branch-site random effects likelihood (BSREL) - a "branch-site" method for detecting the branches on which a proportion of codons evolve with $\omega>1$ (Kosakovsky Pond et al. 2011). MEME is capable of detecting episodic positive selection, especially when these instances are located on a small portion of the tree branches, meaning that it can detect positive selection in the overwhelming presence of negative selection (Murrell et al. 2012). FUBAR is more robust to model mis-specification and also orders of magnitude faster in terms of algorithmic implementation (Murrell et al. 2013). Furthermore, we employed the mechanistic empirical model (MEC) (Doron-Faigenboim and Pupko 2007) that accounts for the different amino acid replacement probabilities based on the JTT empirical substitution matrix (Jones et al. 1992), while estimating the codon rate matrix, thus allowing for positions undergoing radical amino acid exchanges to acquire higher $\mathrm{dN}$ rates than those with less radical exchanges. The codon-wise $\omega$ estimates were mapped onto predicted protein tertiary structures deposited in PDB (http:// www.pdb.org; human prion protein (PrP), PDB ID: 1QLZ; human Doppel protein (Dpl), PDB ID: 1LG4) using the Selecton-3D web server (http://selecton.tau.ac.il).

We examined the possibility of natural selection driving the non-independent evolution of codons and amino acid sites at the intra- and inter-molecular level. We adopted a protein primary structure spatial approach by searching for amino acid residues that show evidence of concerted evolution in CAPS as detailed in (Fares and McNally 2006).
First, we looked for residues that appear to be linked on the protein structure or potentially functionally as a result of natural selection (Fares and Travers 2006) using the PDB tertiary structures. After searching for coevolving sites within proteins, we contrasted the two proteins. Significance was assessed via random resampling of the correlation coefficients for residue pairs sampled from the alignment $(100,000$ pseudoreplicates, $\alpha=0.001)$, and the amino acid sequence distances among taxa was Poissoncorrected. Groups of coevolving residues were set at a maximum of 3-5\% of the total alignment length. Protein structures were plotted in Jmol 13.0 (http://www.jmol.org) and network relationships among coevolved residues were mapped in Cytoscape 3.0 (http://www.cytoscape.org).

\section{Results}

\section{Prion Protein Selection}

Sequencing of 19 novel rodent PRNP sequences yielded a phylogeny generally consistent with species phylogeny as has been previously described for prion sequences (van Rheede et al. 2003). Exceptions were the hedgehog (Erinaceus europaeus) and guinea pig (Cavia porcellus) positions (Fig. 1). It is generally unsurprising that conflicts arise between a gene tree of a sequence under selection does not precisely recapitulate a species tree as would be expected of a neutral marker. A scan for positive diversifying selection using MEME revealed five codons (codons 22, 68, 130, 197, and 220$)$ to belong significantly $(P<0.05)$ to this regime, but once the significance cut-off was reduced to 0.01, codon 197 was dismissed (Fig. 1, Supplementary Table 1). Codon 22 had two inferred nonsynonymous substitutions along the branch leading to the greater kudu (Tragelaphus strepsiceros). Codon 68 had two nonsynonymous substitutions on the internal branch connecting the European rabbit (Oryctolagus cuniculus) with the other Glires. Codon 128 showed similar evidence (1-2.7 nonsynonymous substitutions) on the branches leading to the Malayan colugo (Galeopterus variegatus), the rabbit, the pangolin (Manis sp.), the Assam macaque (Macaca assamensis), and the dog (Canis familiaris). Codon 220 harbored two nonsynonymous substitutions on the branch of the house mouse (Mus musculus M180071). The FUBAR method did not detect any codons under pervasive diversifying selection exceeding a $\operatorname{PP}(\mathrm{d} N>\mathrm{d} S)>0.9$, but at $\mathrm{PP}=0.81$ codons 125 and 127 emerged, although with an expected number of false positives of 0.38 (CI $95 \%$ 0-2). When we searched for episodic positive selection along the branches of the PRNP gene tree, we found small, isolated bursts of diversifying selection acting on a limited number of codons on the internal branch before the Rodentia-Scadentia clade ( $p=0.000055$, Holm's 
Fig. 1 Maximum likelihood phylogenetic tree for $P R N P$. Branches with significant $(p<0.05)$ evidence of episodic positive selection as indicated by the branch-site REL model are marked with star symbols. Sequences obtained from GenBank are shown with accession numbers. All rodent sequences generated in this study are shown without accession numbers. Accession numbers for sequences generated in this study can also be found in the "Materials and Methods" section. The area of the circles at the nodes indicates the magnitude of internode branch support. The scale bar denotes 0.02 nucleotide substitutions per site

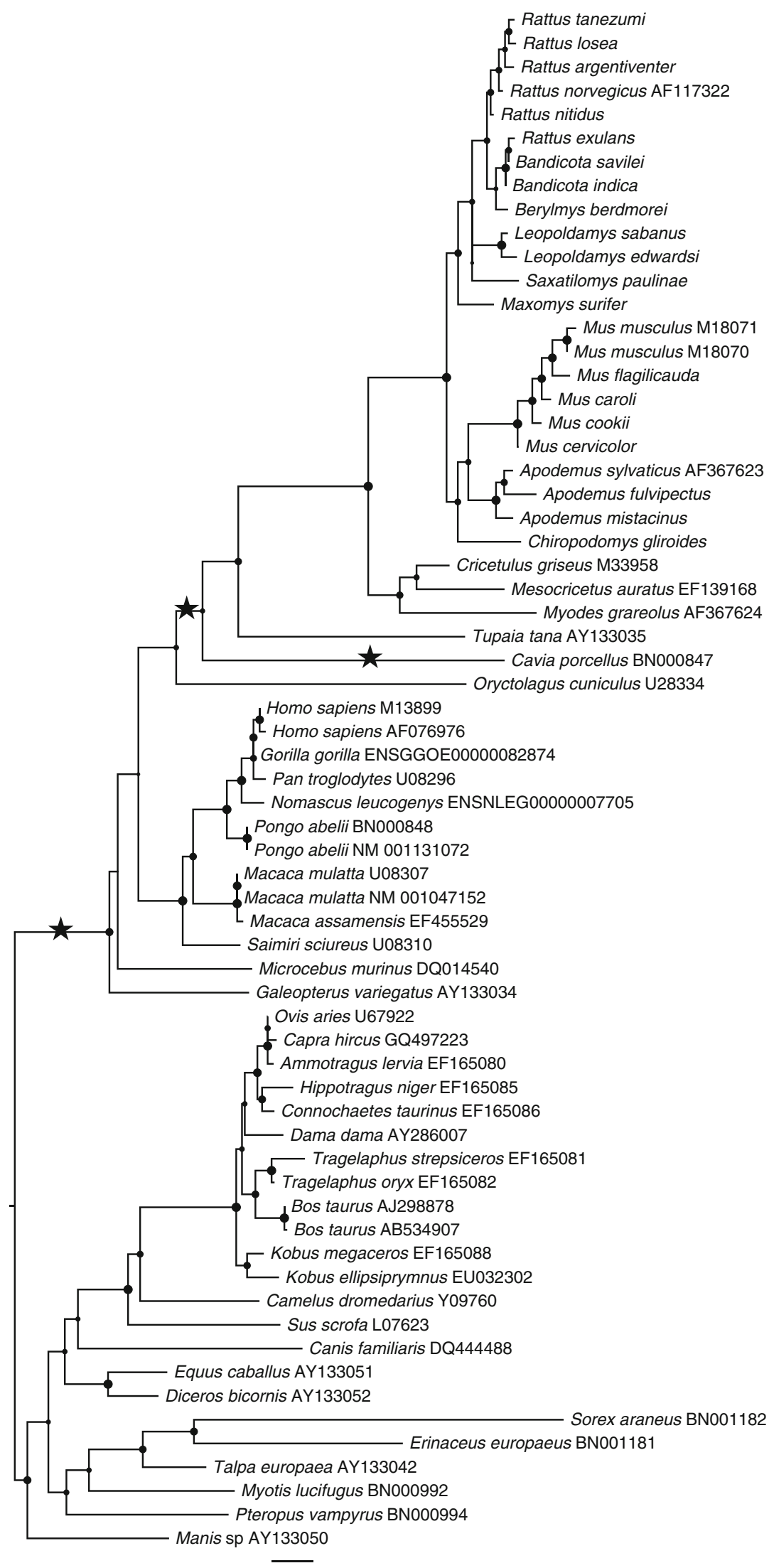

0.02 
Fig. 2 Detection of selective pressure on PrP and Dpl using the mechanistic empirical combination model mapped on protein tertiary structures. The color scale illustrates the intensity of selective constraints. a, b PrP, c, d Dpl. The codon-wise $\omega$ estimates were mapped onto predicted human protein tertiary structures from PDB (PrP: 1QLZ; Doppel: 1LG4). b, d, Black boxes indicate the location of $\beta$-sheets in the protein while, red and blue rectangles illustrate the location of 3/10 helix and $\alpha$-helix structures, respectively
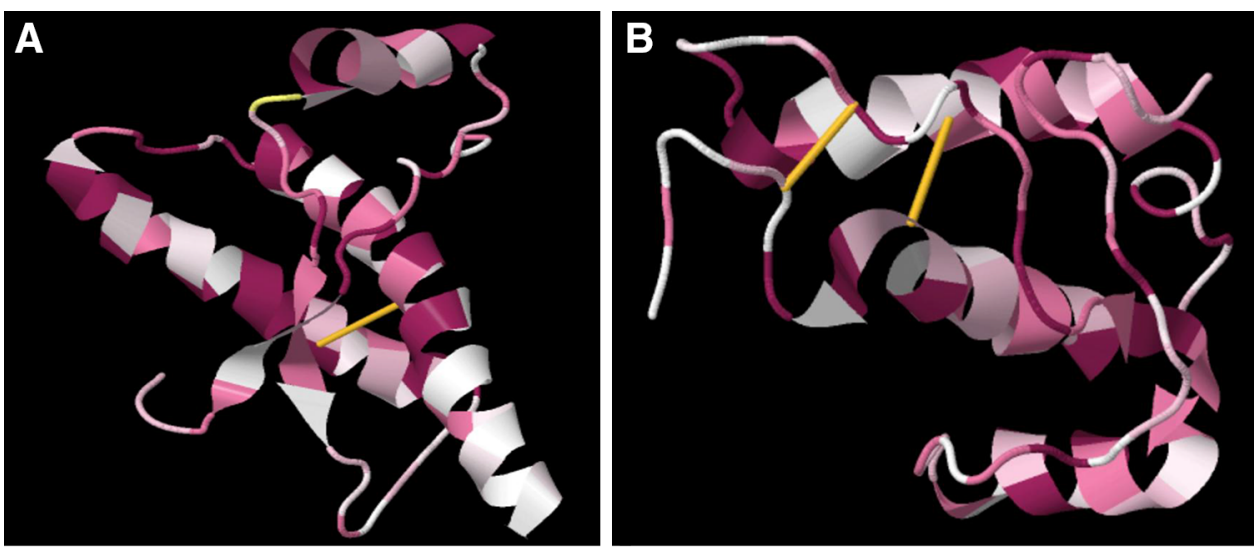

C

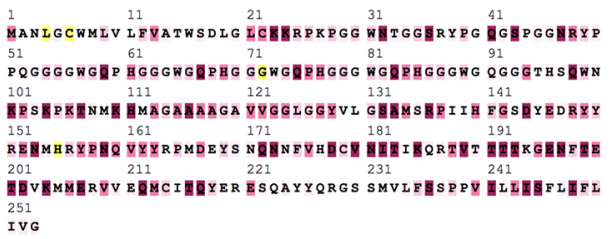

D

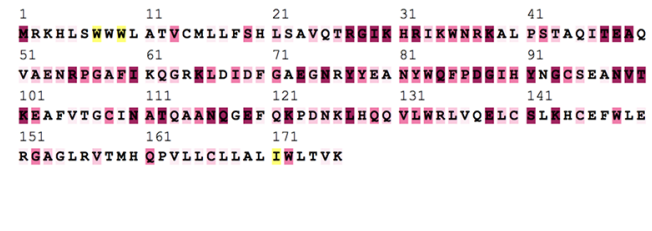

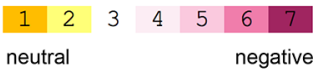

correction for multiple comparisons), the Euarchontoglires ( $p=0.0173$ ), and the guinea pig (Cavia porcellus) branch $(p=0.0358)$. Negative purifying selection was widespread on $P R N P$ present in 185 codons with $\mathrm{PP}(\mathrm{d} S>\mathrm{d} N)>0.9$ of which 157 codons had a PP $>0.95$ and 106 codons had PP $>0.99$ (Supplementary Table 1). When the data of Premzl and Gamulin (2009) were examined alone or in combination with our expanded data set, three codons were found to be under episodic diversifying selection in both data sets (Fig. 1 and data not shown) (Premzl and Gamulin 2009). However, in both analyses, the codons reported by Premzl and Gamulin (2009) were not the same as observed in either of the datasets as analyzed in the current study. When modeled on the tertiary protein structure of human PrP, purifying selection could be observed throughout both the $\alpha$ helices and the $\beta$-sheets that link them (Fig. 2a, b). Relaxation of purifying selection was localized in residues that link the first and second $\alpha$-helical domains.

\section{Doppel Protein Selection}

The PRND phylogenetic analysis included 20 novel rodent sequences and was generally consistent with the species tree with a few deviations, i.e., the hedgehog PRND is sister to Bovidae PRND and the mouse lemur (Microcebus murinus) grouping with the guinea pig, thus exhibiting divergence from the otherwise monophyletic primates, while the rabbit occupies a basal position (Fig. 3). MEME identified four codons with evidence of episodic diversifying selection (codons 5, 11, 16, and 145; $p<0.05$ ).
FUBAR identified codon 145 to be under positive selection $[\operatorname{PP}(\mathrm{d} N>\mathrm{d} S)=0.93$, Supplemental Table 1]. While still pervasive, negative selection was less widespread on $P R N D$ than on $P R N P$ with 92 codons being identified as selected against with $\mathrm{PP}(\mathrm{d} S>\mathrm{d} N)>0.9$, of which 65 codons had a PP $>0.95$, and 30 had a PP $>0.99$ (Fig. 2c, d). The branch-site REL model did not identify any branches, internal, or external, under episodic positive selection.

Prion and Doppel Coevolutionary Trends

When examining inter-molecular coevolutionary trends between the two proteins we found eight PrP residues with putative Dpl interaction sites forming four distinct networks with mostly one-to-many relationships with Dpl sites (Fig. 4). None of the eight PrP sites in our analysis, which show evidence of interactions with Dpl sites, have been linked with any transmissible neurodegenerative diseases identified in humans or other mammals so far (Collinge 2001) (Mastrangelo and Westaway 2001). The majority of putative interactions identified do not appear to have specific patterns among the secondary or tertiary structures of the two proteins with Dpl residue 113 and 114 as the only exceptions. Dpl residue 113 of the $\mathrm{H} 2 \alpha$-helix appears to interact only with $\operatorname{PrP~H} 3 \alpha$-helix residues (222, 224, 225), while Dpl residue 114, also part of the $\mathrm{H} 2 \alpha$ helix has putative interactions only with PrP residue 174 part of the $\mathrm{H} 2 \alpha$-helix of PrP protein (protein) (Riek et al. 1998; Golaniska et al. 2004). 
Fig. 3 Maximum likelihood phylogenetic tree for $P R N D$. Sequences obtained from GenBank are shown with accession numbers. All rodent sequences generated in this study are shown without accession numbers. Accession numbers for sequences generated in this study can also be found in the "Materials and Methods" section. The area of the circles at the nodes indicates the magnitude of internode branch support. The scale bar denotes 0.02 nucleotide substitutions per site

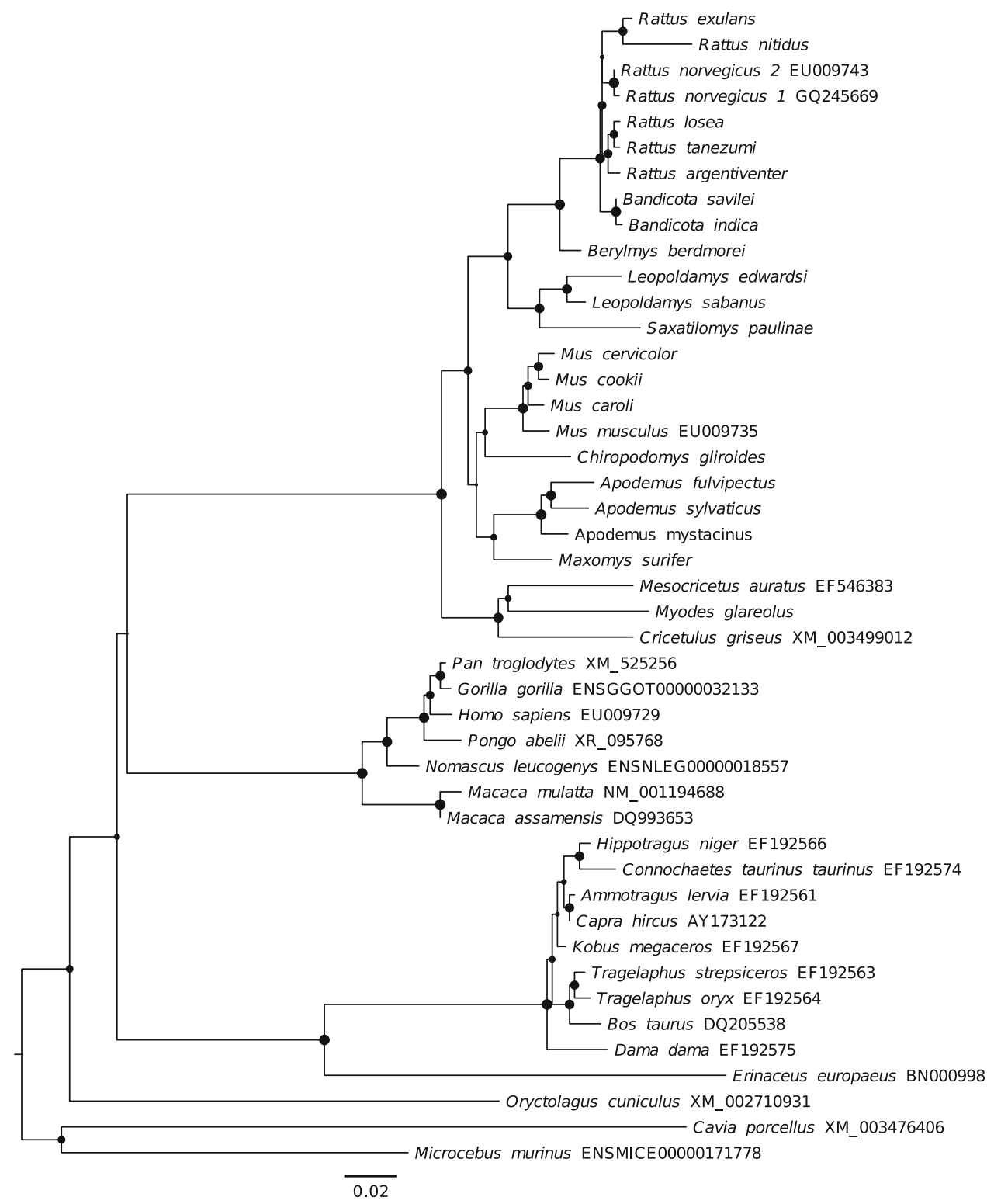

\section{Discussion}

No evidence of balancing selection or widespread positive selection could be identified in our study for either PRNP or $P R N D$. This is consistent with an earlier interspecific study (Krakauer et al. 1998), but at odds with a recent one (Premzl and Gamulin 2009). This latter study examined seven PRNP sequences from representatives of the Euarchonta focusing on primates (five species) as well as twelve sequences from Laurasiatheria (3 Cetartiodactyla, 2 Perissodactyla, 1 Carnivora, 1 Pholidota, 2 Chiroptera, and 3 Eulipotyphla species) and found evidence for positive selection on specific branches and codon sites. Some of those amino acid sites are potentially important for PrP function: His-100-Asn replacement is implicated in cross-primate transmission (Schatzl et al. 1995), and two changes spatially related to the binding site of protein X (Kaneko et al. 1997; Perrier et al. 2002). We could not confirm these specific results for PRNP using the original data set of (Premzl and Gamulin 2009) or by expanding our dataset to 48 taxa and by utilizing more powerful selection detection computational methods, other than a burst of positive selection on the branch leading to the Perissodactyla clade (BSREL, $p=0.04$ ) given the Premzl and Gamulin (2009) sequences alone. Once we added our sequences this result was no longer significant (BSREL, $p=0.147)$. Our contrasting findings with respect to Premzl and Gamulin (2009), who previously reported the action of positive selection on specific codons in PRNP, are very likely due to the taxonomic and-subsequentlysequence variability between their 19-taxon and our 48-taxon datasets. 


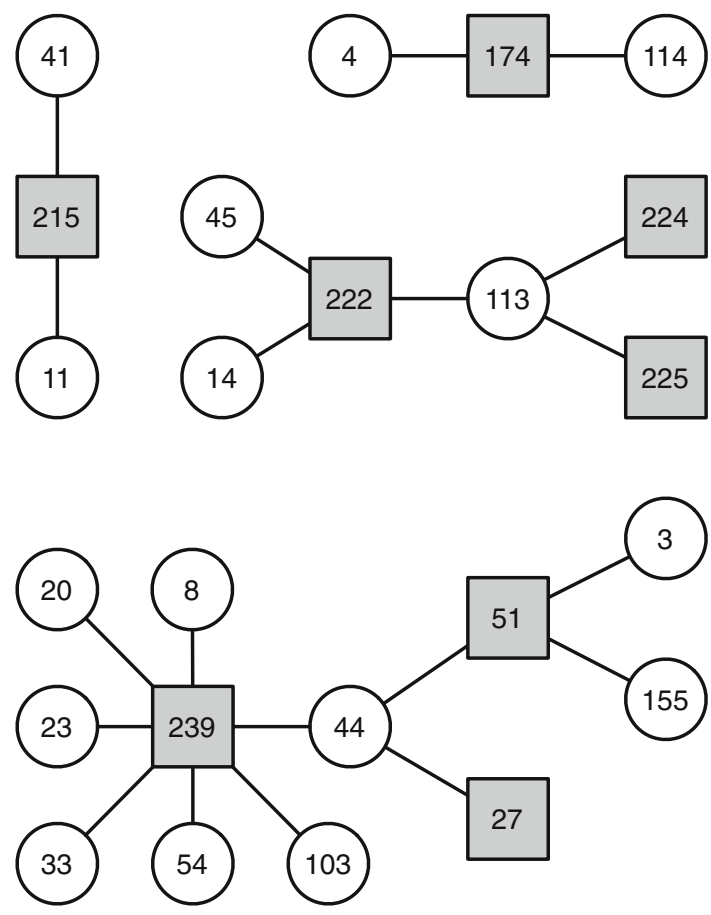

Fig. 4 PrP-Dpl coevolution analysis. Numbers in boxes indicate codons in gray (PrP) and white (Dpl). Four coevolution groups were identified. Network edges denote inferred interprotein functionally interacting amino acid residues

While an earlier study (Mead et al. 2003) reported intense balancing selection against homozygous polymorphisms in humans and concluded on a recurring pattern of balancing selection in PRNP in the latest 500,000 years of human evolution, subsequent studies criticized that claim on the basis of the introduction of a bias in the ascertainment of single nucleotide polymorphisms (SNPs) by selecting and scoring SNPs with frequency $>5 \%$ (Kreitman and Di Rienzo 2004; Soldevila et al. 2006). Resequencing efforts of the PRNP exon 2 and population genetic estimations (Soldevila et al. 2006) were unable to support the balancing selection claim and found evidence suggesting the potentially heterotachous action of positive selection. The presence of some intermediate-frequency polymorphisms remains interesting and warrants a wider resequencing study and examination of the effect of the polymorphisms on protein structure and function. Another study (Seabury et al. 2004) evaluated genetic variation in the exon 3 of PRNP in 36 breeds of domestic cattle and other bovine species and found negative selection against nonsynonymous changes and an excess of rare silent polymorphisms upstream the $\mathrm{N}$-terminal cleavage site coding sequence.

Our results are most consistent with that of Seabury et al. (2004). Although they had a limited interspecific focus using cross-species comparisons in a pairwise fashion examining cattle and bison at the population level with various bovine species as outgroups, their analysis supported purifying selection. Our analysis suggests this and extends it to non-domestic animals as a general principle. Purifying selection was not limited to the Bovidae but extended to all mammal groups studied here, including all primates and the expanded rodent taxa as well. The principle can be generalized to PRNP paralogs such as PRND, which demonstrated a very similar pattern of selection dominated by negative selection in all included taxa. A portion of the constraints may be a result of coevolutionary pressure as several putative interacting sites were detected between PrP and Dpl. Although no interactions were found among residues that correlate with disease and interactions did not strongly correlate with predicted structural motifs, some evidence for interaction between the $\mathrm{H} 2$ and $\mathrm{H} 3 \alpha$ helices of PrP and Dpl were identified. Further experimentation will be necessary to determine the significance of these sites in interaction between the protein products of these two genes.

From a biological standpoint, purifying negative selection could be interpreted as a consequence of the pathogenic effects of misfolding of the prion protein. Selection against changes that could favor the pathogenic conformations would be advantageous. Existing polymorphisms in prion genes can lead to a higher probability of seeding conversion to $\operatorname{PrP}^{\mathrm{Sc}}$ (Christen et al. 2013). It has also been suggested that variants conferring susceptibility to TSEs are in generally conserved regions and that such variation is generally derived (Martin et al. 2009). This also extends to the species barrier to prion diseases whereby most species are resistant to infectious prions of other species. The species barrier may be determined by variation in few amino acids in disordered regions of the prion protein (Richmond et al. 2014). Overall there is strong conservation of the PrP at the sequence and structural levels among diverse species (Richmond et al. 2014; Wopfner et al. 1999). In this context, our result of general negative purifying selection could indicate that variants that confer higher probability of seeding misfolding are quickly removed from the population. However, relaxation of selection at specific domains such as at the amino acids connecting $\alpha$-helices 1 and 2 could suggest that substitutions in these regions do not destabilize the prion protein in such a way as to provoke it into adopting a pathogenic conformation.

Surprisingly, $P R N D$ is under very similar constraints although it is not directly associated with neurological disease. This may reflect either linkage disequilibrium effects as $P R N D$ and $P R N P$ are closely linked or may be due to constraints imposed by the putative interaction between the two proteins identified as coevolving sites in this study. The similar selection regimes on PRNP and $P R N D$ in all taxa examined suggest that purifying selection 
on the prion genes and its homologs may be a general feature for this gene family in all species. This is somewhat surprising as the species tested are not equally susceptible to prion diseases with most species, particularly primates and rodents, being generally resistant to disease but several cervid species quite disease prone including to the emergence of CWD in wild cervids (Saunders et al. 2012). Nonetheless, there was no evidence for relaxed selection in cervids suggesting that their relative susceptibility to prion diseases is related to other factors such as ease of transmission or within species polymorphism (Hunter 2007) as opposed to the general selective regime on the gene.

Rodents have been hypothesized to be potential reservoirs of TSEs (Heisey et al. 2010). Therefore, we specifically extended the PRNP and PRND data sets for diverse rodent species. Consistent with other taxa, rodent $P R N P$ and $P R N D$ were under a strict regime of negative selection. This is consistent with the difficulty of establishing rodentadapted scrapie models which involves intracerebral inoculation. Therefore, we conclude that the prion gene and its homolog doppel have been evolving under purifying selection among mammalian taxa.

Acknowledgments We thank the French ANR Biodiversity, Grant ANR 07 BDIV 012 CERoPath project (Community Ecology of Rodents and their Pathogens in a changing environment (www.cer opath.org), and the French ANR CP\&ES, Grant ANR 11 CPEL 002 BiodivHealthSEA project (Local impacts and perceptions of global changes: Biodiversity and health in Southeast Asia) (www.bio divhealthsea.org) and all the CERoPath participants for their great help in field works. We also thank the Conservation Genetics Unit of the University of Liège and the Belgian Funds for the Scientific Research (FNRS) for supporting Johan Michaux. The provision of samples and support of the investigations by Jona Freise (Oldenburg), Hanan Sheikh Ali (Greifswald-Insel Riems) are kindly acknowledged. We acknowledge the help of Sergei L. Kosakovsky Pond with the programs HyPhy and Spidermonkey.

\section{References}

Abascal F, Zardoya R, Telford MJ (2010) TranslatorX: multiple alignment of nucleotide sequences guided by amino acid translations. Nucleic Acids Res 38:W7-W13

Caputo A, Sarnataro D, Campana V, Costanzo M, Negro A, Sorgato MC, Zurzolo C (2010) Doppel and PrPC co-immunoprecipitate in detergent-resistant membrane domains of epithelial FRT cells. Biochem J 425:341-351

Christen B, Damberger FF, Perez DR, Hornemann S, Wuthrich K (2013) Structural plasticity of the cellular prion protein and implications in health and disease. Proc Natl Acad Sci USA 110:8549-8554

Collinge J (2001) Prion diseases of humans and animals: their causes and molecular basis. Annu Rev Neurosci 24:519-550

Delport W, Poon AF, Frost SD, Kosakovsky Pond SL (2010) Datamonkey 2010: a suite of phylogenetic analysis tools for evolutionary biology. Bioinformatics 26:2455-2457
Doron-Faigenboim A, Pupko T (2007) A combined empirical and mechanistic codon model. Mol Biol Evol 24:388-397

Fares MA, McNally D (2006) CAPS: coevolution analysis using protein sequences. Bioinformatics 22:2821-2822

Fares MA, Travers SA (2006) A novel method for detecting intramolecular coevolution: adding a further dimension to selective constraints analyses. Genetics 173:9-23

Felsenstein J (1981) Evolutionary trees from DNA sequences: a maximum likelihood approach. J Mol Evol 17:368-376

Golaniska E, Flirski M, Liberski PP (2004) Doppel: the prion's double. Folia Neuropathol 42(Suppl A):47-54

Heisey DM, Mickelsen NA, Schneider JR, Johnson CJ, Langenberg JA, Bochsler PN, Keane DP, Barr DJ (2010) Chronic wasting disease (CWD) susceptibility of several North American rodents that are sympatric with cervid CWD epidemics. J Virol 84:210-215

Hunter N (2007) Scrapie: uncertainties, biology and molecular approaches. Biochim Biophys Acta 1772:619-628

Jones DT, Taylor WR, Thornton JM (1992) The rapid generation of mutation data matrices from protein sequences. Comput Appl Biosci 8:275-282

Kaneko K, Zulianello L, Scott M, Cooper CM, Wallace AC, James TL, Cohen FE, Prusiner SB (1997) Evidence for protein X binding to a discontinuous epitope on the cellular prion protein during scrapie prion propagation. Proc Natl Acad Sci USA 94:10069-10074

Katoh K, Standley DM (2013) MAFFT multiple sequence alignment software version 7: improvements in performance and usability. Mol Biol Evol 30:772-780

Kosakovsky Pond SL, Frost SD (2005) Not so different after all: a comparison of methods for detecting amino acid sites under selection. Mol Biol Evol 22:1208-1222

Kosakovsky Pond SL, Murrell B, Fourment M, Frost SD, Delport W, Scheffler K (2011) A random effects branch-site model for detecting episodic diversifying selection. Mol Biol Evol 28:3033-3043

Krakauer DC, Zanotto PM, Pagel M (1998) Prion's progress: patterns and rates of molecular evolution in relation to spongiform disease. J Mol Evol 47:133-145

Kraus A, Groveman BR, Caughey B (2013) Prions and the potential transmissibility of protein misfolding diseases. Annu Rev Microbiol 67:543-564

Kreitman M, Di Rienzo A (2004) Balancing claims for balancing selection. Trends Genet 20:300-304

Lanave C, Preparata G, Saccone C, Serio G (1984) A new method for calculating evolutionary substitution rates. J Mol Evol 20:86-93

Martin R, Gallet PF, Rocha D, Petit D (2009) Polymorphism of the prion protein in mammals: a phylogenetic approach. Recent Pat DNA Gene Seq 3:63-71

Mastrangelo P, Westaway D (2001) The prion gene complex encoding $\operatorname{Pr} \mathrm{P}(\mathrm{C})$ and Doppel: insights from mutational analysis. Gene 275:1-18

Mead S, Stumpf MP, Whitfield J, Beck JA, Poulter M, Campbell T, Uphill JB, Goldstein D, Alpers M, Fisher EM, Collinge J (2003) Balancing selection at the prion protein gene consistent with prehistoric kurulike epidemics. Science 300:640-643

Murrell B, Wertheim JO, Moola S, Weighill T, Scheffler K, Pond SL (2012) Detecting individual sites subject to episodic diversifying selection. PLoS Genet 8:e1002764

Murrell B, Moola S, Mabona A, Weighill T, Sheward D, Pond SLK, Scheffler K (2013) FUBAR: a fast, unconstrained bayesian approximation for inferring selection. Mol Biol Evol 30:1196-1205

Perrier V, Kaneko K, Safar J, Vergara J, Tremblay P, DeArmond SJ, Cohen FE, Prusiner SB, Wallace AC (2002) Dominant-negative inhibition of prion replication in transgenic mice. Proc Natl Acad Sci USA 99:13079-13084 
Pond SL, Frost SD, Muse SV (2005) HyPhy: hypothesis testing using phylogenies. Bioinformatics 21:676-679

Premzl M, Gamulin V (2007) Comparative genomic analysis of prion genes. BMC Genomics 8:1

Premzl M, Gamulin V (2009) Positive selection in prion protein. J Mol Evol 68:205-207

Richmond K, Masterson P, Ortiz JF, Siltberg-Liberles J (2014) Did the prion protein become vulnerable to misfolding after an evolutionary divide and conquer event? J Biomol Struct Dyn 32(7):1074-1084

Riek R, Wider G, Billeter M, Hornemann S, Glockshuber R, Wuthrich K (1998) Prion protein NMR structure and familial human spongiform encephalopathies. Proc Natl Acad Sci USA 95:11667-11672

Sakaguchi S (2008) Antagonistic roles of the N-terminal domain of prion protein to doppel. Prion 2:107-111

Saunders SE, Bartelt-Hunt SL, Bartz JC (2012) Occurrence, transmission, and zoonotic potential of chronic wasting disease. Emerg Infect Dis 18:369-376

Schatzl HM, Da Costa M, Taylor L, Cohen FE, Prusiner SB (1995) Prion protein gene variation among primates. J Mol Biol 245:362-374

Seabury CM, Honeycutt RL, Rooney AP, Halbert ND, Derr JN (2004) Prion protein gene (PRNP) variants and evidence for strong purifying selection in functionally important regions of bovine exon 3. Proc Natl Acad Sci USA 101:15142-15147

Silverman GL, Qin KF, Moore RC, Yang Y, Mastrangelo P, Tremblay $\mathrm{P}$, Prusiner SB, Cohen FE, Westaway D (2000) Doppel is an $N$ - glycosylated, glycosylphosphatidylinositol-anchored proteinexpression in testis and ectopic production in the brains of Prnp(o/o) mice predisposed to Purkinje cell loss. J Biol Chem 275:26834-26841

Soldevila M, Andres AM, Ramirez-Soriano A, Marques-Bonet T, Calafell F, Navarro A, Bertranpetit J (2006) The prion protein gene in humans revisited: lessons from a worldwide resequencing study. Genome Res 16:231-239

Stamatakis A (2006) RAxML-VI-HPC: maximum likelihood-based phylogenetic analyses with thousands of taxa and mixed models. Bioinformatics 22:2688-2690

van Rheede T, Smolenaars MMW, Madsen O, de Jong WW (2003) Molecular evolution of the mammalian prion protein. Mol Biol Evol 20:111-121

Westaway D, Genovesi S, Daude N, Brown R, Lau A, Lee I, Mays CE, Coomaraswamy J, Canine B, Pitstick R, Herbst A, Yang J, Ko KW, Schmitt-Ulms G, Dearmond SJ, McKenzie D, Hood L, Carlson GA (2011) Down-regulation of Shadoo in prion infections traces a pre-clinical event inversely related to $\operatorname{PrP}(\mathrm{Sc})$ accumulation. PLoS Pathog 7:e1002391

Wopfner F, Weidenhofer G, Schneider R, von Brunn A, Gilch S, Schwarz TF, Werner T, Schatzl HM (1999) Analysis of 27 mammalian and 9 avian PrPs reveals high conservation of flexible regions of the prion protein. J Mol Biol 289:1163-1178

Yang Z (1993) Maximum-likelihood estimation of phylogeny from DNA sequences when substitution rates differ over sites. Mol Biol Evol 10:1396-1401 